Call for Papers 2019:

Können wir Sie als Autor gewinnen?

Wirtschaftsinformatik \& Management versteht sich als hochwertige, wissenschaftlich fundierte Fachzeitschrift für den Themenbereich Wirtschaftsinformatik und relevante Aspekte der Unternehmensführung. Der Qualitätsanspruch der inhaltlichen Arbeit wird durch ein hochkarätiges Advisory Board aus Wissenschaft und Praxis gewährleistet. Top-Entscheider und renommierte Fachautoren analysieren, bewerten und schreiben über zukunftsorientierte Themen für Praktiker auf höchstem fachlichen Niveau.

Können wir auch Sie zu den nachstehenden Themen als Autor gewinnen?

\section{Fachbeiträge allgemein}

In jeder Ausgabe finden sich neben den Texten zum Heftschwerpunkt Fachbeiträge zu Themen aus dem gesamten Spektrum der Wirtschaftsinformatik. Bitte senden Sie vorab einen Abstract (maximal eine Seite) an den Chefredakteur peter.pagel@springer.com.

\section{Schwerpunktthemen}

Als Themenheft setzt Wirtschaftsinformatik \& Management in jeder Ausgabe einen inhaltlichen Schwerpunkt. Für folgende Schwerpunktthemen können Sie sich noch mit Ihrem Beitrag an die E-MailAdresse des Chefredakteurs bewerben:

\section{Heft 1 DSVGO - Blick auf die Praxis}

Heft 2 Jubiläumsheft: 10 Jahre WuM

Heft 3 Mensch-Maschine-Kommunikation:

Es geht voran

Heft 4 Agile - nur ein Buzzword?

Heft 5 Digitalisierung in der Dienstleistungsgesellschaft

Heft 6 E-Learning \& Co. - Weiterbildung im

\section{Jahrhundert}

\section{Advisory Board}

Person $\quad$ Organisation

Prof. Dr.

Andrea Back

Universität St. Gallen

Dr. Udo Bub

Deutsche Telekom

Laboratories

Prof. Dr.

Dieter Ehrenberg

Universität Leipzig

Prof. Dr.

Manfred Grauer

Universität Siegen

Dr. Jürgen Laartz

McKinsey \& Company, Inc.

Prof. Dr.

André Ludwig

KLU Hamburg

Prof. Dr. Dr.

h.c. mult.

Peter Mertens

Universität Erlangen-Nürnberg

Prof. Dr. Matthias

Schumann

Universität Göttingen

Prof. Dr.

Udo Winand

Universität Kassel

Dr. Peter Zencke

SAP Deutschland AG \& Co. KG

Wirtschaftsinformatik \& Management verfolgt das Ziel, den Austausch zwischen Theorie und Praxis der Wirtschaftsinformatik zu fördern. Angestrebt ist aus diesem Grund eine ausgewogene Mischung aus forschungsnahen und Beiträgen aus der Praxis. Gemeinsam ist allen Beiträgen der Bezug zur praktischen Arbeit. 